\title{
Study on Software Reliability and Reliability Testing
}

\author{
G. Chandrika1)
}

\begin{abstract}
Software business is on rise today and it has become the half and parcel within the lives of kith and kin. Such software once used as associate application ought to provide the client a feeling of trait that is nothing however technically termed as dependableness. The system should be ready to perform the desired functions in line with the expressed conditions for a selected amount of your time. It is important to take care of the reliability of the software system to stay track on the important data which incorporates resources, capital, details of employees, etc. Software plays associate equal role on with hardware and thus the analysis and measuring area unit done equally. The most challenging task for the business is to develop a reliable software system that consumes a lot of quantity of your time and dear too. The software dependableness will be measured the approaches specifically unit of time between failure and unit of time to failure. In this case we adopt a testing referred to as as dependableness testing whose purpose is to find the potential issues within the phases of development like style as shortly as doable and ultimately give the idea that the system meets its dependableness necessities.
\end{abstract}

Keywords : SMERFS, ATM(Automatic Teller Machine), CASRE., Software, Reliability

\section{Introduction}

The paper aims to provide a study on software reliability and reliability testing. Software has become the backbone of many applications like medical, business, transportation, etc[1][2]. There are many applications existing in the field but the number of users vary for each and every application[3]. There is a matter of reliability that is trustworthiness for any application to become successful[4]. As the software is increasing day by day the problems on size and complexity are also increasing proportionally[5]. In order to get rid of these problems they introduced several reliability models. There are some tools been introduced which help us to measure, track and improve software reliability. There are some user friendly packages like SMERFS and CASRE for managing the reliability. For example consider an application say ATM(Automatic Teller Machine) which is been used by thousands of users daily which perform transactions and say that there is some fault in the application like software or

Received(January 12, 2015), Review Result(1st: January 23, 2015, 2nd: February 10, 2015), Accepted(March 10, 2015)

${ }^{1}$ (Corresponding Author) Vidyaa Vikas Educational Institutions, Mysore - Bannur Road, Alanahally, Mysuru, Karnataka, India

email: gudechandrika@gmail.com 
hardware like scanner or credit card reader is not working then the situation is very disastrous and all the customers will be frustrated. There should be some best practices and tools needed to overcome these mistakes or problems. So in 1990's they initiated to grade and promote the quality of a software which is measured in terms of Software Engineering Institutes(SEI) Capability Maturity Model(CMM) levels. SEI rates the particular product in terms of levels(0 to 5), Initial process capability(level 1), Optimizing process capability(level 5). If the level is more then the success rate of the product is more. Lower the CMM level more faults are anticipated and therefore there will be lower the predicted reliability on that particular product.

\section{Problem}

The problem or issue being solved or described here is as follows some reliability growth models are applied while testing the products. One of the most important models used id dynamic failure data. In 1980 's there are 50 to 80 models are produced. There are sufficient models developed by the researchers to test the reliability of the product but no guidelines are available which would have helped the practitioners to test their own product. Moreover the invention of many models created confusion among the developers. To come across this problem American Institute of Aeronautics and Astronautics (AIAA) recommended four growth models which include

1) Schneidewind model

2)JelinskiIMoranda model

3)Musa/Okumoto logarithmic Poisson execution-time

4)Littlewood-Verrall model.

When the developed product is tested through these models several factors like test strategies, error detection and handling, effectiveness, quality, etc are tested which helps to deploy a more reliable product. SRE activities perform different tasks at various phases.

\section{SOFTWARE RELIABILITY MODELLING}

There need to be some tools developed to make SRE techniques more accessible to the practitioners to test their products. The models are complicated to compute and therefore there is a lot of effort placed to develop the tools. Tools are used to provide estimates initial failure intensities and also s-confidence intervals around these estimates. The tools will be taking 
interval and time domains as input data.

\subsection{SMERFS TOOL}

Which is abbreviated as Statistical Modelling and Estimation of Reliability Functions for Software. This is the first tool developed in the year 1983 which implements a wide variety of software reliability models. This tool is menu driven interface which is designed for easy use. The functionalities are defined as following data entry, editing, and transformation model application $>$ determination of model applicability CASRE TOOL: Which stands for Computer Aided Software Reliability Estimation tool. This tool is developed to measure the software reliability. This incorporates the mathematical modelling capability of the public domain. CASRE tool address many issues like ease of use and many more. It is the extension of SMERFS and a portable tool which is indented to work in any environment. There is a menu driven command interface and several different models will be executed in this environment and then the predicted failure intensities are drawn.

The non homogeneous Poisson process based software reliability growth model for N-version programming systems based on the non homogeneous Poisson process. Due to continuous extraction of errors from the software versions the people don't consider growth of reliability in N-version programming systems. A debugging effort is used to remove the errors or faults during the debugging and testing the product. New errors and faults may be introduced instead of removing them successfully due the complexity of the system.

The new N-VP software reliability growth model is established in which the multi version failures which are coincident modeled by applying the generalized non homogeneous Poisson process (NHPP) model into N-version programming (NVP) system. The application of this new software reliability can be illustrated by estimation of system reliability they are provided with the s-confidence bound.

To predict the Nvp systems performance and for the evaluation of the reliability this model can be used. The N-vp SRGM is also used for overcoming the short comes of reliability model which is independent. The systems reliability can be predicted much accurately than any model which is independent and can also be used to determine when the testing should be stopped. It is a key question in N-vp SDLC (software development life cycle).

There are independent and common faults. Faults cannot be removed successfully and new faults are introduced into the system while debugging process. The system reliability cannot grow if the faults are introduced in the debugging process which cannot be done in function of intensity failure. In NVP system different faults in that system has different roles. 
It can be tolerated successfully if it is S-independent. If the faults are common for multiple versions then they will be activated with the same input then multiple versions will fail.

Using various languages and various philosophies there will some effect on software reliability. Common fault is situated in the modules of functional equivalent in 2 versions of software.

The programmers will do same errors or mistakes even though different versions are developed by them. The fault type of the common failures is common.

The output of it is usually same. The fault location is also same. The voting result in common failure will choose the wrong solution.

The conclusion reached on the basis of the paper are how the data of a particular model is being tested and which standard method it is following. Various types of reliability growth models are being tested. The software reliability growth model assumes testing can be performed randomly or homogeneously. With the help of some mechanism which is random the data of test can be chosen from the input and the testing of the software can be done assuming the conditions which are homogeneous. The number of test runs will be conducted during the phase of testing. The input is given in such a way that testing will be effective and we can recognize more faults. If any failure has occurred then many test runs will be conducted to avoid it or to reduce it. Clusters occur when failures have a chance to occur in group which is also known as cluster.

The system fails when automatically two versions of the software fails. The mechanism of decision is perfect as assumed and there are two categories as discussed above that is common modes of failure and S-independent modes. Due to software faults the system will fail while execution.

The failures of the software follow NHPP. Many SRGM which are dependent on NHPP became more successful tools in SRE i.e. software reliability engineering.

The failure of the software rate is directly proportional to the no. of remaining faults in the software by that time. Due to software failure the effort of debugging occurs immediately which will remove the corresponding faults.

The solution, outcome, or resolution of the problem or issue is as follows

Both discrete and continuous time of SRM models can be constructed. With the failure independence the input and time domain models are derived. It is a flexible model of reliability.

There are 2 stages in it:

1. Discrete time can be constructed with the help of software runs. 2. Continuous time can 
be constructed with the execution time of the software.

Adaptable model for both s-dependent and independent sequential runs in a software.

Applicable to various phases of SDL (software development life cycle).

Poisson process is the simple one and consider only failure points such as among 2 failures the successive runs are disregarded and the information told by them will be ignored. If there is a failure in some runs then with the removal of the faults the probability of the occurrence of the faults will change. When the failure occurs the faults should be recognized and removed immediately. This is an important approach for more consistent SR(software reliability) modelling.

Many reliable software models are developed to help the managers and developers to analyse the process of developing software for cost estimation and to know the software reliability levels. The NHPP model is applied to design the failure process of software among all others models of reliable software. It is also used to analyze when to stop the testing and when to release it. The NHPP models of software reliability are taken out with function of intensity rate in the faults in the stable or controlled environment.

The success or failure depends on the previous test runs output. Every software takes some time for execution. The s-dependent trail of Bernoulli is also known as sequence of test runs conducted by a software. For both success and failure runs the execution time of the software is not distributed identically.

The biases or assumptions behind the inferences, selection or collection of data, or framing of the problem is

Many models of software reliability are the extension of the jelinskimoranda model. There are some assumptions to be taken in this model:

1. There will be some initial faults in the code or a program which is not known to us but it is a fixed or may be random sometimes. 2. Each and every faults are independent in the program and will mostly cause the same failures while testing. 3. For every occurrences of failure the time intervals are not dependent on each other. 4. The faults are removed immediately whenever there is an occurrence of failure. 5. The faults which are responsible for the failures should be removed instantly. 6. Again the other faults should not be introduced after the detected faults removal. 7. The failure rate of the software is fixed while the interval of the failure. 8. It is also equal to the number of remaining faults in a program.

The basic concepts or terms used are

Reliability: The trustworthiness or quality that can be dependable on a particular product.

Capability maturity model(CMM): The degree of optimisation and formality of a product 
that aims to improve existing software product.

Software reliability growth models (SRGM's): Fundamental technique for quantitative assessment of software reliability.

Maximum likelihood estimation (MLE): The method of estimating the parameters of statistical model of a given data.

Non-Homogeneous Poisson Process (NHPP): Similar to poisson process but the average rate of arrivals can be varied with time.

Software development life cycle (SDLC):It is series of phases or steps which provides a model for the developing and managing a piece of software.

Fault: Lack of correctness in code that causes failure.

Failure: When the external behaviour of a particular product is incorrect it is defined as failure.

Imperfect debugging: If a fault is detected and not eliminated permanently during the stage of debugging is defined as Imperfect debugging.

\subsection{Failure datasets}

This section lists several application data sets that, throughout the book, can be used to implement and illustrate the software reliability modeling.

Data Set \#1: On-line Data Entry Software Package Test Data The small on-line data entry software package test data, available since 1980 in Japan[6], (Data set \#1). The size of the software has approximately 40,000 LOC. The testing time was measured on the basis of the number of shifts spent running test cases and analyzing the results. The pairs of the observation time and the cumulative number of faults detected.

Data Set \#2: On-line Communication System (OCS) The On-line Communication System (OCS) project at ABC Software Company was completed in 2000[7]. The project consisted of one unit-manager,

Testing time 123456789101112131415161718192021

Failures 21112222173122441613131

Cumilative failures $23 \quad 457911 \quad 1219212224263031373841424546$

one user interface software engineer, and ten software engineers/testers. The overall effort for each of the four phases in the software development process of the project can be described as follows:

Phase 
The data was collected over a period of 12 weeks during which time the testing started and stopped many times. Errors detection is broken down into subcategories to help the development and testing team to sort and solve the most critical Modification Requests (MRs) first. These sub-categories are referred to as the severity level depending on the nature of the problem with 1 being the most severe problem, with 2 being the major problem and 3 being a minor problem. The data set \#2, maps into week, consists of three types of errors: severe 1, severe 2, and severe 3 . The observation time (week) and the number of errors detected per week are presented as follows.

OCS Failure Data (Data Set \#2)

\begin{tabular}{|c|c|c|c|c|c|c|c|}
\hline Week & Severe1 & Severe2 & Severe3 1 & & 4 & 7 & 102 \\
\hline 1 & 5 & 23 & 0 & 0 & 44 & 1 & 4 \\
\hline 65 & 1 & 4 & 66 & 10 & 15 & 87 & 4 \\
\hline 6 & 48 & 1 & 5 & 39 & 1 & 1 & 110 \\
\hline 3 & 7 & 6 & 11 & 0 & 0 & 112 & 0 \\
\hline
\end{tabular}

Date Set \#3: Failure Data from Misra[10]. A set of failure data taken from Misra, given in Table 4.7, consists of three types of errors: critical, major, and minor. The observation time (week) and the number of failure detected per week are shown as belows.

Major errors

Minor errors 1622.60692444 .40243406 .101743212 .5116

52418.9035618190137720 .10228521 .303591223 .8024109024 .1200 2117625.3034125426 .8017133228 .9030148929 .50005156730 .430231645 31.2053172337 .505318244024192949 .502420515002

Data Set \#4: US service plan of action information Systems (NTDS) The code information set, listed in Table 4.8, was extracted from information concerning failures in the development of code for the time period multi-computer advanced of the America service Fleet programing Center of the America service plan of action information Systems (NTDS). The software consists of thirty eight completely different project modules. The time horizon is divided into four phases: production phase, test phase, user phase, and subsequent take a look at part. The 26 code failures were found throughout the production part, five throughout the take a look at part and; the last failure was found on four Jan 1971. One failure was observed throughout the user part, in September 1971, and two failures throughout the take a look at part in 1971. 
Data Set \#5: bicycle-built-for-two Computers code information Project This set of unleash \#1 failure information, given in Table 4.9, is from one of four major releases of software product at bicycle-built-for-two Computers (Wood 1996).

information Set \#6: On-Line Data Entry IBM code Package The information according by Ohba (1984a) area unit recorded from testing associate degree on-line information entry code package developed at IBM. Table 4.10 shows the try of the observation time (days) and the accumulative range of errors that were detected.

Data Set \#7: AT\&amp;T System $T$ Project The AT\&amp;T's System $T$ is a network-management system developed by AT\&amp; T that receives information from measurement events, such as alarms, facility-performance information, and diagnostic messages, and forwards them to operators for further action. The system has been tested and failure data has been collected. Table 4.11 shows the failures and the inter-failure yet as accumulative failure times (in CPU units).

Data Set \#8: time period management Systems, the software for monitor and time period management systems consists of concerning two hundred modules and every module has, on average, 1000 lines of a high-level language like FORTRAN. The records the code failures detected throughout the 111-day testing amount. This actual data is cuplike overall with many up and downs reflective completely different clusters of detected faults.

Standard read of independent agency code assurance

Software dependability consists of three main steps that is followed by the life cycle of the code. Those area unit the steps that are given in the on top of diagram. As we apprehend that the method of code has supported the first and $2 \mathrm{~d}$ steps. Now we tend to would like to review the technique of third step.

Here we see the models that can tell this scenario for the dependability of the code and might observe the conditions of the long run work. It is a difficult task to model the failing method of the code.

The reliability models area unit largely used for getting the code quality and to rectify the issues the mandatory resources should be bought. These all steps are done in the upkeep part of the life cycle method. The variables of the software area unit not dependent.

SR model Taxonomy

SR models are divided into two models:

Static model : This is also known as as settled model.

Dynamic model: This is also known as probabilistic model.

The static models uses the resources from the program rather than form the info that is 
didn't analyse the failures.

To find the speed of the dependent variables the freelance variables area unit calculated for this product. Here in this model the quantity of the products within the code is measured for his or her existence.

To develop the profile of the software system some models that area unit reliable can analyse the failing information obtained by the computer programme.They area unit statistically distributed that are referred to as dynamic additionally.

Static versus dynamic reliability model

There are two known models:

1. Halsted's software metric model two. McCabe's cyclomatic complexity model

Now the dynamic models area unit divided into half-dozen elements. They are as follows:

1. Seeding of errors. 2. Growth of reliability three. Fitness of curve 4 . Rating of failures 5. Poisson process that is Non homogenous half-dozen. Structure of Markov

These are some of the strategies used for the estimation of the dependability of the code. The complexity, safety, remaining errors can additionally be measured within the code.

Static SR model (Deterministic SR model)

To measure the software complexity Halsted's model is one of the best models. The difficulty levels in testing as well as debugging the software can also be reduced with this model. McCabe's model is used for the representation of the control flow in the program.

Halstead's software metric model:

$$
\begin{array}{ll}
N=N 1+N 2 & V=N \log 2(n 1+n 2) \\
N 1=n 1 \log 2 n 1 & N 2=n 2 \log 2 n 2
\end{array}
$$

Where

$n 1=$ number of different operators

$n 2=$ number of different operands

$N 1=$ number of occurance of the operators

$N 2=$ number of occurrences of the operands

$N=$ program length

$V=$ volume of the program

$E=$ errors in the program

$I=$ number of instructions in the machine

To find the defects in the program Halstead introduced 2 formulaes:

$$
\dot{\mathrm{E}}=\mathrm{V} / 3000
$$




$$
\grave{\mathrm{E}}=\mathrm{A} / 3000
$$

where

$$
A=(V /(2 n 2 / n 1 N 2))^{\wedge} 2 / 3
$$

$V=$ assembly language volume

McCabe's cyclomatic complexity:

$$
V(G)=e-n+2 p
$$

Where

$e=$ number of edges in the control graph

$n=$ number of vertices in the control graph

$p=$ connected elements in the graph

The multiple graphs which are cyclomatic is equal to the its sum.

$$
F P=e-n+2
$$

Where

$n=$ total nodes

$e=$ total edges

The edges are denoted with arrows and nodes are denoted with circles in the above diagram.

Enhanced version of growth model in web based SR engineering:

To improve the reliability of the software for big systems is the task of the enhanced version. The customers will expect more reliability of the application of the system.

In distributed software the reliability of the web application is very important case. This kind of software is distributed over the network. Detection of the failures in the hardware is much easier than detection of failures in the software.

Software Reliability which is web based:

When talking about the web based SR we need to consider many technologies. Each application will be having some failures such as some delays and the system can be unreliable. Web consists of html pages, servlets, some other sources etc.

Every model consists of

\section{Client 2. Server 3. Database}

Every architecture consists of n-tier based design pattern. The hardware and software mistakes are considered in the global analysis.

Reliability means the number of faults the software or the system contains and it is its functioning. To become more useful the data is taken from the growth model which id deployed. 
When the product is into the market then number of failures in the software collected is more as it is distributed to various at a time. The number of failures obtained is divided into units. When there are $\mathrm{N}$ software installations and $\mathrm{F}$ failures at time $\mathrm{T}$ then the software failure rate is given by

$$
\lambda=F /\left(N^{*} T\right)
$$

When the failures occurs then there is a loss of time that is because it also needs the time for repair of the faults. Joining the repair time with the mean time we can judge that for how much time the software is not available.

MTBF is the mean time between the failure

$$
M T B F=M T T F+M T T R
$$

It can be derived easily with the observation on the growth of the reliability. Its parameters have an advantage in the process of testing.

\subsection{Exponential approach:}

This model consists of 2 parameters. It is obtained randomly. The distribution function for every $\mathrm{i}$ can be given as, $F i(t i)=1-e-\lambda i$

For calculating the average:

The mean time can be calculated with this.

\subsection{Enhanced exponential approach:}

On the basis of the failure information the present status of the failure can be calculated. This is not based on the initial stage of the system.This can detect the failures accurately.The test cases are executed and the number of the defects are caught and blocked. The defects are also made open at the time of phase of the release of the software.

This is a procedure of inference for the calculation of the prediction is given as follows:

The priority of the model is not considered in the growth model. The severity of the model is also not considered in the growth model. The detection of the coming defects is obtained. Whenever the faults are recognized then they should be rectified. Based on the last information the next faults are rectified.

It is not suitable for the web applications. some web apps requires some APIs which have some different modules and some different users.

\subsection{SR improving with the software engineering approach}


There are some activities to be followed for the improvement. They are as follows:

Construction: The new documents and code for the program is generated.

Preparation: The test plan and cases will be operated for the output that is for the execution.

Combination: The old and new documents are combined and reused.

Correction: The errors will be identified and removed with the use of static method of analysis.

Testing: Whenever the failure occurs then cases for test are executed.

Repair: The errors are banished.

Validation: It is done to check if the methods used for execution are effective or not.

Retesting: To check whether all the tests are executed without errors or not.

Metric process

With the help of the metric process the reliability of the product can be improved.

Product metrics

The software is written in different language. The functionality of the software is measured. When the function is recognized then with is help the size also can be measured.

There are some graphs related to reliability. They are as follows:

1. Bathtub curve 2. Software reliability curve 3. Size and complexity curve

The tasks which undergo in this process are:

1. Constructing 2. Combining 3. Correcting 4. Preparing 5. Testing 6. Identifying the errors 7. Repairing 8. Validating 9. Retesting

There are some metrics in Project management. With the proper project management task the best product can be built according to the knowledge of the researches.

This leads to proper time management and the best quality of the product. There is an important thing that if we use different approaches which are not reliable then there will be increase in the cost for building the product.

The flexibility, Interoperability and maintainability must be taken utmost care.

1. The flexibility is nothing but the work required for transferring the code from one hardware to hardware.

2. The work required for joining one system with another system is nothing but the interoperability process.

3. Maintainability means the work done should be modified without having any errors and the analysis, Testing should also be done.

For the high quality software development there are engineering approaches. They are:

1. SE TOOLS 2. SE METHODS 3. SE PROCESS 4. QUALITY PRODUCT 
The high quality software development is shown in the form of a diagram.

These are nothing but the techniques for the better improvement of the software.

The strategy for the testing is as follows and it contains the system, validation, combined and unit testing.

The process that takes place in for the reliability of the software is as follows

In requirement analysis there area unit a lot of errors found as a result of it is terribly troublesome for human action and analysing the code. This was also told by the researches.

1. The requirements should be known properly.

2. Software needs area unit higher than the hardware needs.

3. The proper prototypes should be developed.

4. The requirements ought to be studied properly.

5. With the help of the flow diagrams the analysis of the structure should be done.

6. At the starting itself we tend to ought to bear in mind of your time that wants for the completion of the building of the merchandise.

7. The cost required for completion of the merchandise should even be taken care.

8. The effort needed for the merchandise should even be analysed within the early stages.

9. The management of the risks should be done properly.

\section{Conclusion}

Software business is on rise these days and it has become the half and parcel within the lives of folks. Such software once used as Associate in Nursing application ought to provide the client a feeling of trustiness that is nothing however technically termed as responsibleness. The system should be in a position to perform the desired functions in keeping with the declared conditions for a particular amount of your time. It is vital|vital|important $\}$ to keep up the reliability of the code to stay track on the important data which has resources, capital, details of employees, etc. Software plays Associate in Nursing equal role on with hardware and so the analysis and mensuration area unit done equally. The most challenging task for the business is to develop a reliable code that consumes a lot of quantity of your time and pricey too. The software responsibleness will be measured the approaches particularly mean solar time between failure and mean solar time to failure. In this case we adopt a testing known as as responsibleness testing whose purpose is to find the potential issues within the phases of development like style as shortly as doable and ultimately give the idea that the system meets its responsibleness needs. We have additionally learned totally different growth models and the 
way a specific project is calculable. Reliability plays a terribly crucial role in developing a specific project and usage of correct growth model helps in obtaining a trustworthy implementation.

\section{References}

[1] A. Avizienis and L. Chen, On the implementation of $\mathrm{N}$-version programming for software fault-tolerance during program execution, Proc. COMPASAC., (1977), pp.149-155.

[2] K. Kanoun, M. B. Martini, J. M. de Souza, A method for software reliability analysis and prediction: Application to the TR.OPIC0-R switching system, IEEE Trans. Software Engineering, (1991), pp334-344.

[3] L. Chen and A. Avizienis, N-version programming: A fault tolerance approach to the reliable software, Proc. 8th Int. Symp. Fault-Tolerance Computing, Toulouse., (1978), pp.3-9.

[4] Software Reliability Estimation and Prediction Handbook. (1922), AIAA

[5] S. Yamada and S. Osaki, Software reliability growth modelling Models and applications, IEEE Trans. Softw. Eng., (1985) December, Vol.SE-11, No.12, pp.1431-1437.

[6] S, Yamada, M. Ohba and S. Osaki, s-Shaped Software Reliability Growth Models and Their Applications, IEEE Transactions on Reliability, (1984), Vol.R-33, No.4, pp.289-292.

[7] S. M. Resnick, D. L. Pham, M. A. Kraut, A. B. Zonderman and C. Davatzikos, Longitudinal Magnetic Resonance Imaging Studies of Older Adults: A Shrinking Brain, ournal of Neuroscience, (2003), Vol.23, No.8, pp.3295-3301.

[8] Carnegie Mellon University, https://users.ece.cmu.edu, June 11 (2014).

[9] ReliaSoft, http://reliasoft.com/, June 11 (2014).

[10] P. N. Misra, Software reliability analysis, IBM Systems Journal, (1983), Vol.22, No.3, pp.262-270. 